\title{
Revisiting quark stars under the influence of strong magnetic fields
}

\section{Debora Menezes}

\author{
Universidade Federal de Santa Catarina, Brazil
}

email: debora.p.m@ufsc.br

\begin{abstract}
Quark matter at finite temperature and subject to strong magnetic fields is possibly present in the early stages of heavy ion collisions and in the interior of protoneutron stars. We use the mean field approximation to investigate this type of quark matter described by the Nambu-Jona-Lasinio model. The energy per baryon of magnetized quark matter becomes more bound than nuclear matter made of iron nuclei, for magnetic fields around $10^{19} \mathrm{G}$. When the $\mathrm{su}(3)$ NJL model is applied to stellar matter, the maximum mass configurations are always above 1.45 solar masses and may be as high as 1.9 solar masses for a central magnetic field of $10^{18} \mathrm{G}$. These numbers are within the masses of observed neutron stars but exclude the recently measured star with 1.97 solar mass.

The effect of the magnetic field on the effective quark masses and chemical potentials is only felt for quite strong magnetic fields, above $5 \times 10^{18} \mathrm{G}$, with larger effects for the lower densities. Spin polarizations are more sensitive to weaker magnetic fields and are larger for lower temperatures and lower densities.
\end{abstract}

